\title{
Sputtering During Microanalysis in the Analytical Electron Microscope
}

Nestor Zaluzec

Argonne National Laboratory, Bolingbrook, Illinois, United States

Energy transfer by incident electrons occurs to all materials whenever they are studied in electron microscopes. If the energy of the electron beam is sufficient, atoms may be displaced from their lattice sites; either to form point defects, which may subsequently migrate and cause elemental rearrangement, or they can be sputtered from the specimen. This effect is manifest by a variety of events which include: structural damage via radiolysis and/or atomic displacement [1-3]; charging and heating [4-5]; mass loss by sputtering or gain by contamination [6-7] as well as by elemental redistribution during microanalysis [8-9]. In all cases, the amount of kinetic energy transferred ( $\mathrm{T}$ T) by an electron (mass $=\mathrm{m}_{0}$ ) to the nucleus of a specimen depends upon the kinetic energy of the incident electron probe $\left(\mathrm{T}_{0}=\mathrm{eV}_{0}\right.$, e=electronic charge $\& \mathrm{~V}_{0}=$ accelerating potential), the mass of the target nucleus $(\mathrm{M})$ and the direction of scattering $\varphi$ ) of the electron beam. This can be written as:

$$
T_{T}=\frac{\left[2 T_{0}\left(T_{0}+2 m_{0} c^{2}\right)\right]}{M c^{2}} \sin ^{2}\left(\frac{\phi}{2}\right)
$$

with the forward scattering direction defined as $\varphi=0^{\circ}$. This dependence as a function of atomic number for various accelerating voltages is plotted in Figure $1 \mathrm{~A} \& \mathrm{~B}$, for forward $\left(\varphi=0^{\circ}\right)$ scattering.

Beyond some critical energy the energy transferred to the atoms within the solid is sufficient to either sputter $\left(T_{S}\right)$ from the surface of the solid or to permanently displace $\left(T_{d}\right)$ an atom from its lattice site. It is this point at which electron damage becomes important to microanalysis since the atoms in the solid no longer are constrained and can become mobile or are completely removed from the specimen, thus changing the local composition.

Experimental values of $T_{d}$ or $T_{s}$ are not extensive, however, it has been proposed that these should be related to the sublimation energy ( $\mathrm{TSub}_{\mathrm{Su}}$ ) of an element by a multiplicative factor. This factor ranges from 1.5-2.5 for surface sputtering and 3-5 for lattice displacements [7,10]. Complicating this simple proposition is the fact that $\mathrm{T}_{\text {sub }}$ in crystalline materials is also a function of crystallographic direction. In FCC metals, the lowest energy directions are generally along the $<110>$ direction while the highest energy are usually perpendicular to the $<111>$. In BCC metals the lowest energy directions tend to be < $100>$ while $<111>$ is generally the highest. In figure $1 \mathrm{C}$ we plot the average sublimation energy of the pure elements, ignoring orientation effects. Using these values of sublimation energy one can solve to estimate the threshold accelerating voltage for sputtering and displacement. This is plotted in figure $2 \mathrm{D}$ for sputtering $\left(\mathrm{T}_{\mathrm{S}} \sim 1.5 \mathrm{~T}_{\text {sub }}\right)$ and displacement $\left(\mathrm{T}_{\mathrm{d}} \sim 3 \mathrm{~T}_{\text {sub }}\right)$. In many cases for materials with $\mathrm{Z}<40$ this threshold value is in the range of $100-200 \mathrm{kV}$, which is a common voltage used in today's instruments. Compounds, oxides and alloys will, of course, have different values of sublimation energy than that of the pure elements which are plotted in figure $1 \mathrm{C}$, and can be lower than of the pure elements. For example the nominal sublimination energy of $\mathrm{Zn}$ metal is $\sim 1.6 \mathrm{eV}$, while $\mathrm{ZnO}$ and $\mathrm{Zn} 2 \mathrm{O}$ are $\sim 0.69$ and $\sim 1.3 \mathrm{eV}$ respectively.

In the past, due mainly to the effects of hydrocarbon contamination, sputtering was only observed in ultrahigh vacuum instruments. Today, the improved vacuum systems and plasma cleaning technology 
[11] has mitigated hydrocarbon contamination, this contamination in effect suppressed sputtering by acting as a replenishing surface barrier. In addition, the propensity of high brightness, high current density electron probes and "clean" analytical systems has created situations where sputtering of lower sublimation energy materials is now often observed. In figure 2, we illustrate this by time resolved hyperspectral imaging of crushed $\mathrm{ZnO}$ crystals which are supported on graphene coated/microporous SiNx films (figure 2A). Here the nominal beam current was $180 \mathrm{pA}$, for a $200 \mathrm{kV}$ electron probe in a ThermoFisher Talos F20, equipped with a Super X Quad SDD detector. Hyperspectral images were acquired over a 450x650 pixel region of interest, at 100 microseconds/pixel for 400 frames (i.e. a total of $\sim 40 \mathrm{msec} / \mathrm{pixel}$ ). The temporally resolved change in microstructure due to sputtering can be clearly seen in figure $2 \mathrm{~B}$, while the change in local composition ratio of the $\mathrm{Zn} / / \mathrm{OK}_{\mathrm{K}}$ and $\mathrm{Zn} K / \mathrm{O}_{K}$ emission as a function of time is shown in figure $2 \mathrm{C}[12]$.
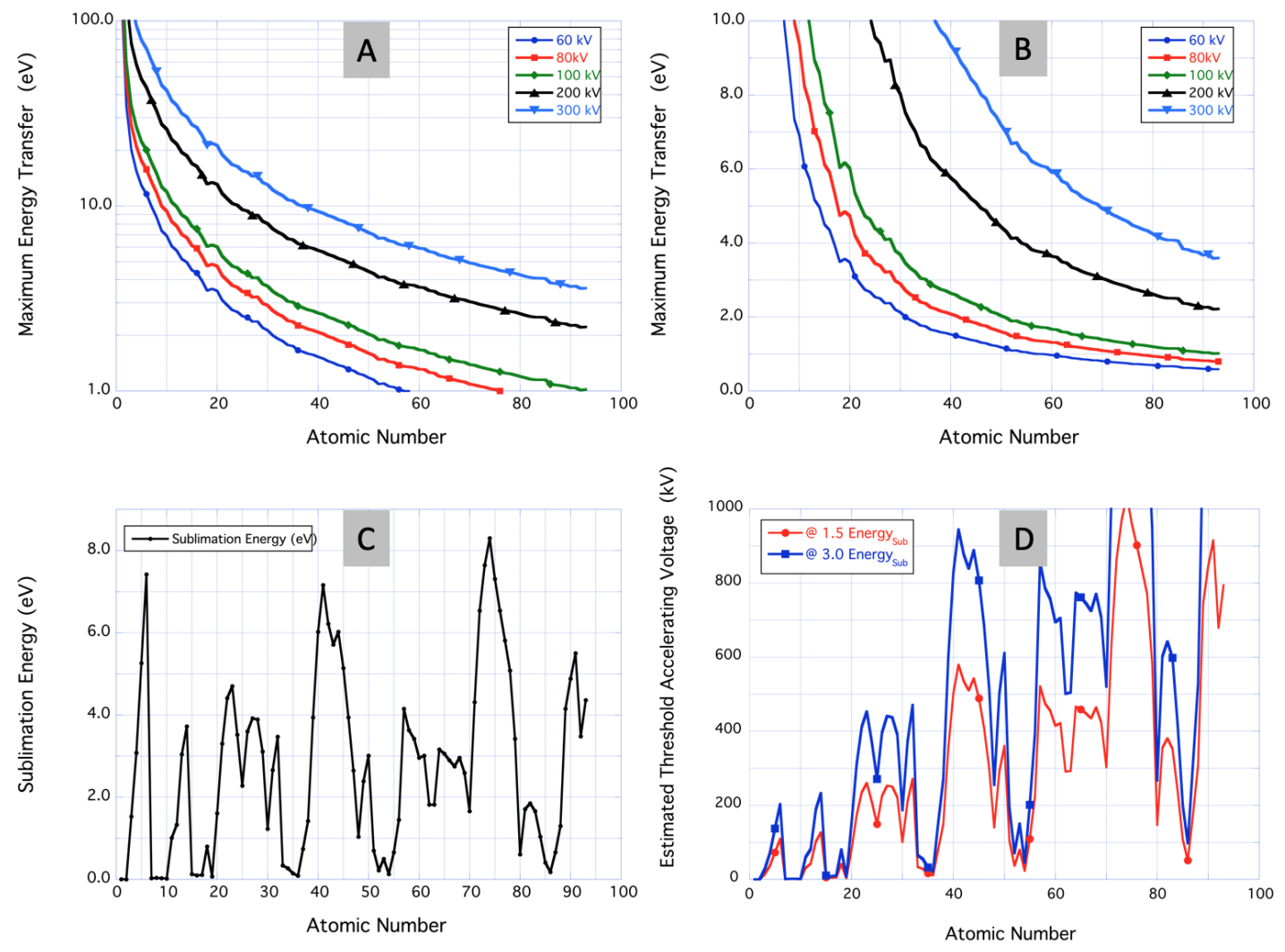

Figure 1. A). Maximum energy transfer $\left(\varphi=0^{\circ}\right)$ as a function of atomic number for $60-300 \mathrm{kV}$ electrons (log scale); B). same plot but using a clipped linear scale. C). Average sublimation energy of the pure elements as function of atomic number. D) Estimated threshold energy for atomic sputtering and displacement as a function of atomic number for thresholds at 1.5 and 3 times the sublimation energy. 

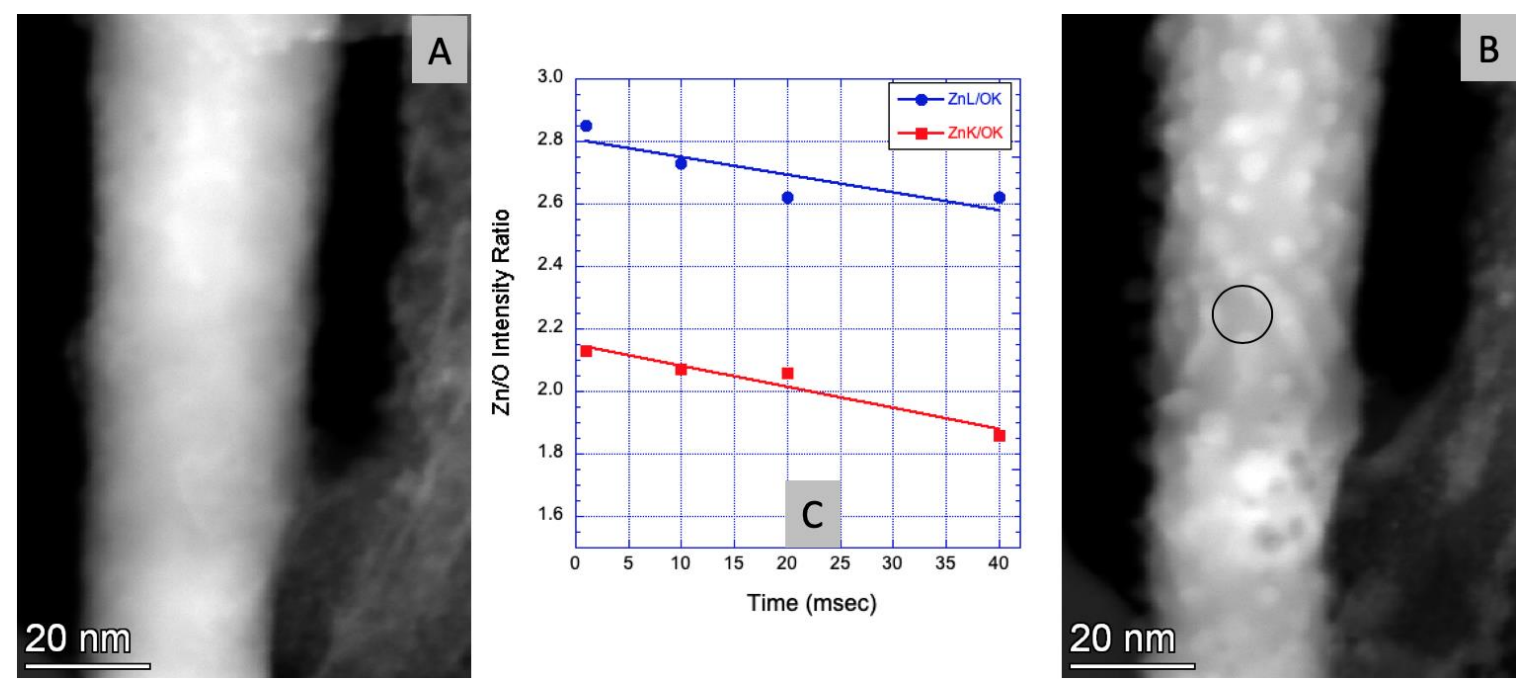

Figure 2. Experimental sputtering effects during time resolved hyperspectral imaging of crushed $\mathrm{ZnO}$ on multi-layer graphene on microporous SiNx. Accelerating voltage $200 \mathrm{kV}$, beam current $\sim 180 \mathrm{pA}$, dwell time 100 usec/pixel over 0-400 frames. A) start, B) after 200 frames C) Integrated intensity ratio of $\mathrm{ZnL} / \mathrm{OK}$ and $\mathrm{ZnK} / \mathrm{OK}$ intensity ratio as a function of time. Data plotted in C) were analyzed from the region of interested marked with at the circled zone.

\section{References}

[1] Isaacson M. (1977) in Principles and Techniques of Electron Microscopy V 7, 1-78

[2] Downing K.H. and Glaeser R.M. (1996), Ultramicroscopy 20, 269,

[3] Medlin, DL, and Howitt DG (1991) Microbeam Analysis (1991) SanFrancisco Press 272-272

[4] Cazaux J Ultramicroscopy (1995), 60,441-425

[5] Egerton R.F. etal, Micron (2004) V35, 399-409

[6] Hren J. (1979) in Introduction to Analytical Electron Microscopy, Plenum Press 481-505

[7] Bradley CR and Zaluzec NJ , Ultramicroscopy (1989) 28, 335-338

[8] Mansfield J.M. etal Ultramicroscopy (1987) 21, 13-22

[9] Massover W.H. etal Micro. Microanal. (2012) 18 S2 1212-1213

[10] F. Seitz Discuss. Faraday Soc. 5, (1949), 571

[11] Zaluzec N.J in Progress in Transmission Electron Microscopy 1: Concepts and Techniques, ( X. F. Zhang, Z. Zhang eds) Springer-Verlag New York, Chapter 10, Pages 343-351, (2001)

[12] This research was supported by the Photon Science Division and employed resources in the Center for Nanoscale Materials, an Office of Science user facility both of the U.S. Department of Energy, Office of Basic Energy Sciences under contract No. DE-AC02- 06CH11357 at Argonne National Laboratory . 